\title{
Optimal Operation of District Heating Networks Through Demand Response
}

\author{
M. Capone, E. Guelpa, V. Verda* \\ Energy Department, Politecnico di Torino, Turin, Italy \\ E-mail: martina.capone@polito.it, elisa.guelpa@polito.it, vittorio.verda@polito.it \\ Received 29 January 2019, Accepted 26 February 2019
}

\begin{abstract}
In this paper, an optimization method aiming at minimizing the thermal peaks in district heating networks is proposed. The method relies on a thermo-fluid dynamic model of both the supply and return networks and permits to analyze the opportunities for thermal peak shaving through "virtual storage". The latter is obtained through variation of the thermal request profiles of the users. The presence of a peak in the morning is due to the shut-down or attenuation of the heating systems during the night, which lead to a dramatical increase of the thermal request early in the morning. The peak compromises a full exploitation of cogeneration and renewable plants that are able to cover just a portion of the maximum load. Consequently, boilers have to be used, leading the system to a performance reduction and to an increase of primary energy consumption. Moreover, the peak makes the possibility of network extension quite difficult, because of the limitation on mass flow rates in the pipes. For this reason, a model is developed to make the thermal profile as flat as possible. The model is applied to a portion of the Turin district heating network, which is the largest network in Italy. Results show that reductions between $20 \%$ and $42 \%$ are possible, depending on the maximum changes in the possible schedules.
\end{abstract}

Keywords: Thermal network model; peak shaving; thermal request variation; optimization.

\section{Introduction}

District Heating (DH) systems provide a reliable and competitive energy service, which is able to satisfy the thermal demands of urban areas and to reduce primary energy consumptions, responding to the main European energy policy objectives. Indeed, the fundamental idea of district heating is to collect heat produced in one or more thermal plants and convey it to the end users through a capillary network of insulated pipes. In particular, DH uses excess heat from combined heat and power (CHP) plants, waste-to-energy plants ( $\mathrm{WtE})$, waste heat from industrial processes [1], as well as heat from renewable sources like geothermal wells, solar collectors and biomass fuels [2].

Thanks to the large number of benefits that district heating technology can boast, such as higher security of supply, lower costs and lower carbon dioxide and pollutant emissions, the future prospects for district heating technology are expected to be promising [2].

Within this framework, full control of thermodynamic parameters assumes a key role. Indeed, the knowledge of mass flow rates flowing in the system and of the evolution of pressures and temperatures in some crucial points is essential to reduce heat losses and minimize the production cost while ensuring the comfort of end-users in buildings [3].

The evaluation of these quantities could be done by equipping the network with proper instrumentation. However, this solution is typically too expensive due to the large cost of sensors and to the intrinsically intrusive nature of this method. Therefore, an appropriate model is needed to forecast the thermo-fluid dynamic behavior of the network. Moreover, modelling the network leads to the possibility to analyze different conditions than the operating ones and various layout, and it permits to evaluate the effects of the changes without the need to carry out expensive and not always replicable experimental tests.

In such scenario, modelling of district heating systems has been widely exploited for both design [4] and management [5] purposes. Among the design applications, modelling can be used for the network dimensioning, as done by Koiv et al. [6] that proposes a method based on the probabilistic determination of the flow rate. Also, a multiobjective optimization model for the best design of a network, taking into account both initial investment for pipes and pumping costs for water distribution, is performed in the work performed by Wang et al. [7]. Modelling is also useful to analyze the possibility of connecting additional users to an existing district heating network, as examined in [8].

Concerning management applications, models are used to develop strategies aiming at reducing primary energy consumptions in operation. Various papers deal with the supply temperature optimization, permitting to reduce distribution losses $[9,10]$. A model for optimizing integration of boilers, heat pumps and cogeneration is proposed by Lindenberger et al. [11].

An important issue in district heating system management, especially in Mediterranean regions, is the presence of a peak request in the morning. The peak is due to the fact that in this area the heating systems are typically switched off or attenuated during the night, leading to a dramatical increase of the thermal request early in the morning. Peaks cannot be completely covered through high efficiency plants (e.g. cogeneration plants), since these are typically sized to cover just a portion of the maximum load (Figure 1). Furthermore the presence of a peak generally involves larger water mass flow rates circulating in the network. This causes higher pumping costs and compromises the possibility of a network extension, since 
water velocity cannot exceed a limit because of structural constraints. The opportunity of installing storage tanks that can be charged when the request is low (i.e. during the night) and used when the request is high (i.e. during the start-up transient) would smooth this effect. This is analyzed by Verda and Colella [12]. A different option for thermal peak shaving consists in virtual storage [13] that is obtained through variation of the thermal request profiles of some users with the goal of producing an effect similar to that obtained using storage. Once the thermal request forecast is done [14], the optimization of the start-up schedule leads to a reduction of the morning thermal request peak and limits the overuse of peak generators, as examined in [15-17].

In the papers [12-17], the optimization procedure only models the return network, while it relies on experimental temperature values for the supply network. This assumption was justified by small changes in the schedules which were considered. In the present paper, both the supply and return networks are modelled in order to obtain more accurate results, also when large changes are considered. The effects of such modelling strategy are examined in terms of accuracy and computational time.

The optimization tool used is described in section 2, while sections 3 and 4 present the application to a subnetwork of the Turin district heating network and the corresponding results. In particular, the validation by comparison of numerical results with the experimental temperature values measured by sensors installed in the substations are first presented. The maximum peak, achieved through application of the optimization when only the return network is simulated, is compared with that obtained with the optimization including both the supply and return networks. Results prove that the proposed tool allows one to improve the results in terms of peak reduction.

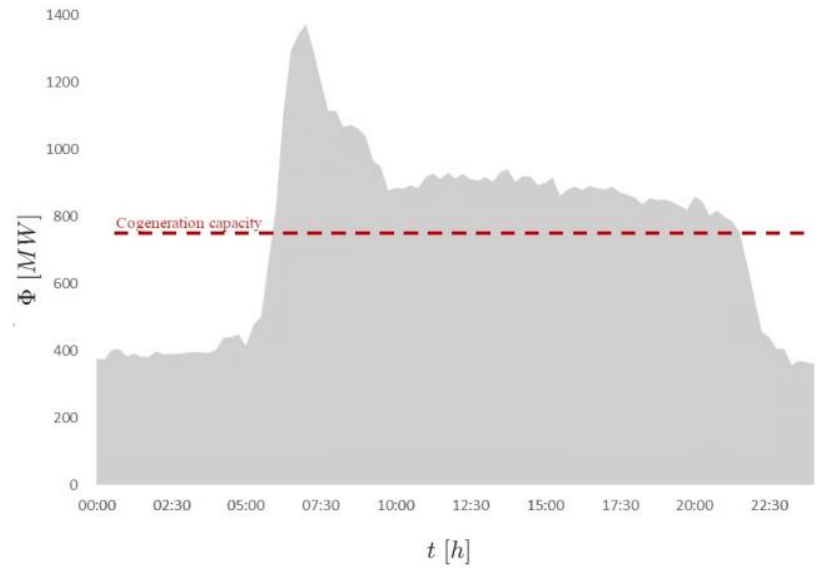

Figure 1 Total thermal load of the coldest winter day in 2017, Turin district heating network

\section{Methodology}

\subsection{Optimal Peak Shaving}

In this work, an optimization tool aiming at minimizing the thermal peak request of district heating networks is presented. This purpose is reached by operating a variation on the thermal request profiles of the users connected with the network.

The decision variable is a vector $\mathrm{x}_{d}$, whose length is equal to the number of buildings, that contains the optimal anticipation time for each heating system. These values can be only discrete. They can be chosen every $5 \mathrm{~min}$, between the lower boundary of 0 min (i.e. no anticipation) and an upper boundary (30 $\mathrm{min}$ in the base optimization case). The anticipations act on the thermal profile and on the mass flow rate request of each building, which are actually the free variables of the optimization problem.

The objective function is represented by the maximum of the heat flux request of the distribution network:

$O . F .=\max \left(\Phi_{t h, t o t}\right)$

where

$\Phi_{t h, t o t}(t)=G_{t o t, B C T}(t) c_{p}\left(T_{\text {supply }}-T_{\text {return }, B C T}(t)\right)$

$G_{t o t, B C T}$ is the total mass flow rate circulating in the network, $T_{\text {supply }}$ is the supply temperature (equal to $120^{\circ} \mathrm{C}$ in the presented application), and $T_{\text {return,BCT }}$ is the temperature of water exiting the distribution network and it is given by the solution of the thermal fluid-dynamic model of the return network.

A genetic algorithm, set for integer values, is used to perform the optimization. It is a stochastic, population-based algorithm, that mimics the selection mechanisms of natural genetic to search for the minimum. The selected population number and the number of generations are set to 100 in this work, and a preliminary analysis has been performed to prove the suitability of these criteria. In particular, the convergence of the algorithm with these parameters has been verified for a simple optimization problem including only the return network simulation on a rough non-refined grid (Figure 2).

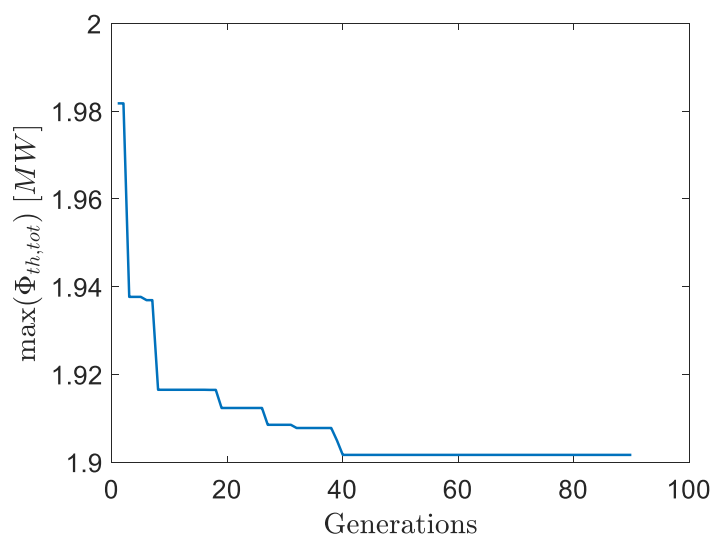

Figure 2 Convergence analysis: objective function value at different generations.

The optimization procedure proposed in this work presents a crucial difference with respect to the one used in previous works on virtual storage. In these works only the return network is simulated, while the temperature values reaching the users are taken by experimental measures and imposed as inputs, by simply operating a translation in case of schedule variation.

Here an innovative approach to solve the optimization problem is proposed: differently from [13], also the supply network is inserted inside the optimization procedure. The rationale behind this choice is to accurately model the dynamics of the network, even when large schedule modifications are applied.

Indeed, when the building demand is varied, the simple translation of the standard-case temperatures introduces an approximation for two main interconnected reasons: 

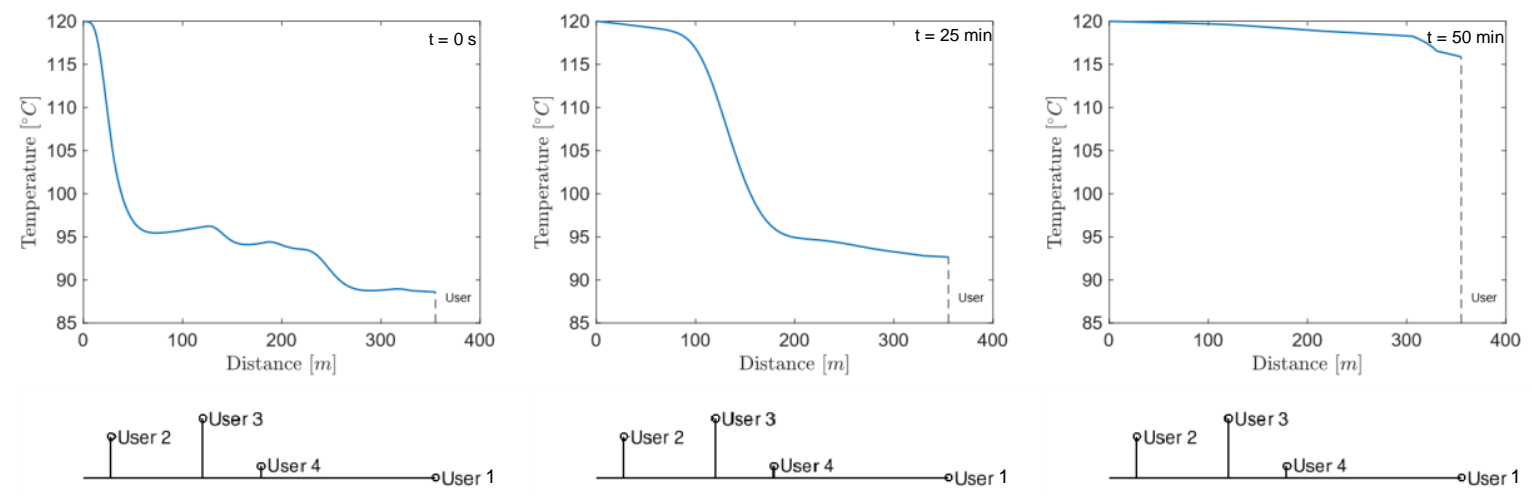

Figure 3. Temperature distribution along the supply network at different time shots.

- a generic building would encounter a different network condition while anticipating its start-up transient. Before hot water reaches the building location, the heat exchanger can be exposed to a "train" of cold water which is different than that in the base case. As an example, Figure 3 shows the supply temperature in a portion of distribution network at three different time instants: $\mathrm{t}=0$, when the heating system of a building is switched on (user 1); $\mathrm{t}=25 \mathrm{~min} ; \mathrm{t}=50 \mathrm{~min}$. During the entire period the heating systems of the other buildings are not activated. At $\mathrm{t}=0$, the left end, where the distribution network is connected with the transport network, the temperature is at $120{ }^{\circ} \mathrm{C}$ (which is the temperature in the transport network). The remaining part of the network is at a temperature between $95^{\circ} \mathrm{C}$ and $90{ }^{\circ} \mathrm{C}$, because of the effects of thermal losses during the night. After 25 minutes, about $100 \mathrm{~m}$ of network have reached about $118{ }^{\circ} \mathrm{C}$, which means that if users 2 and 3 switch on the heating system at this time, they will experience the nominal temperature with a short delay. If no other building switches the heating system on, user 1 needs about 50 minutes before the thermal substation is able to operate in nominal conditions and, thus, exchange the nominal heat flux. Before that time, the heat exchanger operates at partial load.

- the distribution losses are no more the same of the base case, since they depend on the mass flow rate circulating in the system.

These effects are particularly relevant when anticipations are large. Thus, there is a need to simulate also the supply network, in order to have a correct evaluation of the temperatures reaching the buildings, the temperatures in the return network and, consequently, of the total heat fluxes exchanged at the producer and consumers sides.

To appreciate the improvement guaranteed by the procedure here illustrated, a comparison of the peak reductions that can be obtained with the two methods is performed. The two procedures are summarized in Figure 4. In the first approach, the schedules are modified within an optimization framework; the supply network is not simulated and the temperature of water entering the buildings is evaluated using experimental values. These are not modified even if schedules are changed. The return network is calculated in order to obtain the temperature of water leaving the distribution network and, thus, the total heat load supplied to the network. In the second approach, also the supply network is simulated, which permits to improve the accuracy of numerical results, but it doubles the computational time requested for optimization.

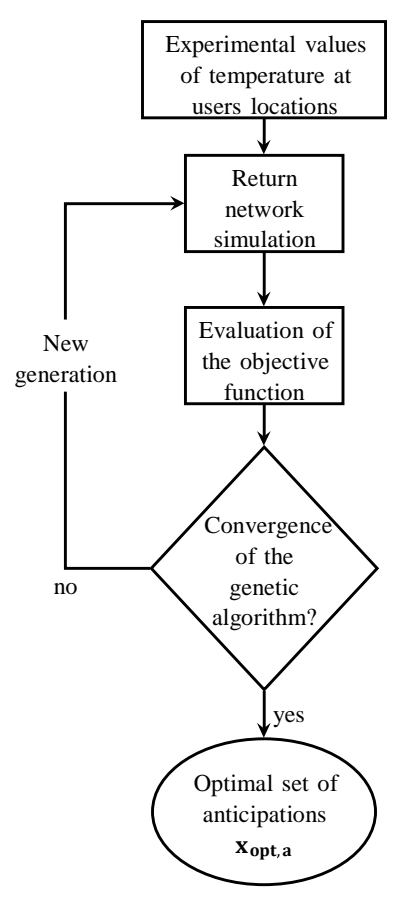

(a)

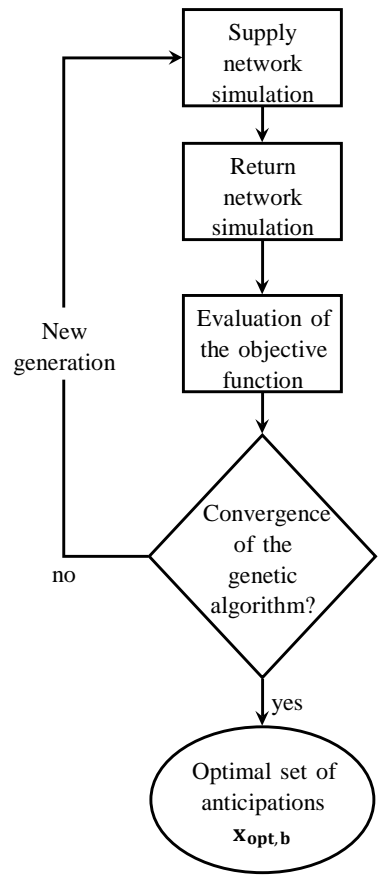

(b)
Figure 4 Schematic comparison of the optimization procedures. (a): Previous works; (b): New optimization procedure

\subsection{Thermal Fluid-dynamic Model}

The supply and return network simulations relies on a thermo-fluid dynamic model, which is based on the conservation equations. The model is pseudo-dynamic [18]: the unsteady term is neglected in continuity and momentum equations, since the fluid-dynamic perturbations linked to velocity and pressure are transferred to the whole network in a period of time of few seconds, which is much smaller than the time step adopted for calculations. Oppositely, energy conservation equation is solved dynamically, since temperature perturbations travel at the fluid velocity, which is typically of the order of few meters per second, and their effect are slowly transferred to the network.

The model is one-dimensional and the complex structure of the network, constituted by pipes, which are connected each other through junctions, is described by means of the graph theory [19]. Each pipe is treated as a branch that starts from a node, representing the inlet section, and ends in another node, the outlet section. The network topology is described by means of the incidence matrix A, which has as 
many rows as the number of nodes and as many columns as the number of branches. A general element $A_{i j}$ is equal to 1 if the $i$-th node is the inlet node of the $j$-th branch, or it is equal to -1 if the $i$-th node is the outlet node of the $j$-th branch. Otherwise, the $i$-th node and the $j$-th branch are not related and the element is equal to 0 .

The fluid-dynamic model is constituted by the integration of continuity and momentum equations, described by Eq. (3) and Eq. (4):

$\frac{\partial \rho}{\partial t}+\frac{\partial(\rho v)}{\partial x}=0$

$\rho \frac{\partial v}{\partial t}+\rho v \frac{\partial v}{\partial x}=\frac{\partial p}{\partial x}-F_{F R I C T}+F_{1}$

where $F_{\text {FRICT }}$ takes into account the viscous forces, and $F_{l}$, representing a source term, accounts for the effect of local fluid dynamic resistance due to valves or junctions and the effects of pressure rise due to pumps.

The continuity equation is applied to all the nodes of the network and integrated over control volumes including each junction node and half of the branch entering or exiting it. Instead, integration of momentum equation is performed over control volumes including a branch and the two delimiting nodes. These equations are respectively expressed by Eq. (5) and Eq. (6).

$\mathrm{A} \cdot \mathrm{G}+\mathrm{G}_{\mathrm{ext}}=0$

$\mathrm{G}=\mathrm{Y} \cdot \mathrm{A}^{\mathrm{T}} \cdot \mathrm{P}+\mathrm{Y} \cdot \tau$

The vector $\mathrm{G}_{\text {ext }}$ contains the mass flow rates which are injected and extracted in the nodes. When the supply network is modelled, this term includes the flow rates injected at the plants and extracted in the substations of the various buildings, and the opposite in the case of the return network. When the heating systems in the buildings do not operate the corresponding term in $\mathrm{G}_{\text {ext }}$ is zero and it is different than zero (positive on the supply network and negative on the return network) when the system operates. The free variables in the optimization process, i.e. the anticipations, act on the time this term is turned from zero to a non-zero value.

In a complex network including loops, an algorithm is needed to solve the problem. A complete description of the method used to solve the fluid-dynamic problem can be found in Sciacovelli et al. [20]. In case of three-shaped networks or subnetworks, mass flow rates can be simply obtained by the resolution of Eq. (5), since mass flow rates distribution is independent of pressure distribution in this case. When loop networks are considered, as iterative algorithm must be considered, as shown in the reference paper.

The thermal problem is expressed by the energy conservation equation. Neglecting the contribution of the conduction along the network, it reads:

$\frac{\partial\left(\rho c_{p} T\right)}{\partial t}+\frac{\partial\left(\rho c_{p} v T\right)}{\partial x}=-\varphi_{l}$

The energy equation is applied in integral form to all the control volumes of the domain. Each control volume includes the junction node and half of each duct entering or exiting the junction, as depicted in Figure 5. Adiabatic and perfect mixing is assumed when different streams converge

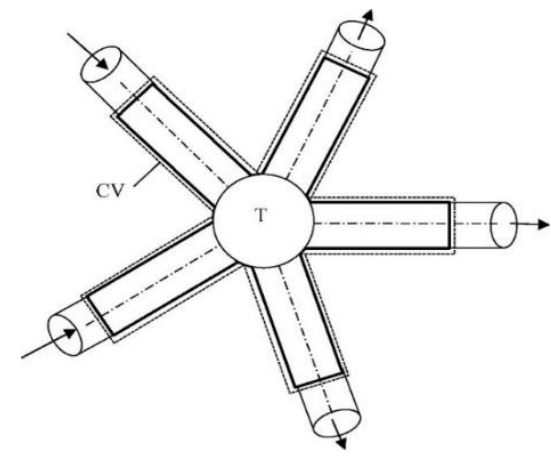

\section{Figure 5. Schematic of the control volume of a junction node [20].}

in a junction: the temperature of all the flows exiting from the junction are at the same temperature.

Integrating Eq. (7) over a control volume $i$ brings to:

$\frac{\partial\left(\rho c_{p} T_{i}\right)}{\partial t} V_{i}+\sum_{j=1}^{N B} \pm c_{p} G_{j} T_{j}=\Phi_{l, i}$

The heat losses $\Phi_{l}$ are ascribed to each branch and they can be calculated considering for the whole control volume the temperature of the node:

$\Phi_{l, i}=\sum_{j=1}^{N B} \frac{L_{j}}{2} \Omega_{j} U_{j}\left(T_{i}-T_{\infty}\right)$

One can observe from Eq. (8) that there is a need to define the temperature $T_{j}$ associated with each boundary of the control volume considered, i.e. the temperature of the middle point of each branch. In particular, it must be expressed as a function of the nodal values of temperature $T_{i}$, which are the unknowns of the problem. Then, the problem can be written for all nodes in matrix form as follows:

$\mathrm{M} \cdot \dot{\mathrm{T}}+\mathrm{K} \cdot \mathrm{T}=\mathrm{g}$

After having applied proper boundary conditions, the problem can be solved.

In Guelpa et al. [21,22], an upwind scheme, that assigns to the cell face the temperature of the upstream node, has been used. This scheme is the simplest scheme that guarantees a stability of the solution, thanks to the fact that it takes into account the direction of the fluid flow. However, it is a scheme of first order accuracy. This means that the truncation error scales with the second derivative of the solution, in a similar manner to the diffusion term, introducing in the problem an artificial-numerical diffusivity which is physically not present and affecting the reliability of the model.

It is possible to prove that the form of this numerical diffusivity is [23]:

$\Gamma_{n u m}=\frac{v \Delta x}{2}$

Then, one way to minimize its contribution is to increase the degree of geometrical discretization. For this reason, it has been chosen to use in the model an advanced upwind scheme: to have a better approximation of the solution, additional nodes, which have a numerical role only, are introduced. In the simulations presented hereafter, nodes are placed with a granularity of 1 node per meter. 


\section{Application}

In this section, a simulation of the thermo-fluid dynamic behaviour of a distribution network of the Turin district heating network, which is the largest DHN in Italy and one of the largest in Europe, is carried out. This system satisfies the energy request of more than 5000 buildings, which have a total volume of about 56 million $\mathrm{m}^{3}$.

The network can be represented as made of two interconnected parts: the transport network and the distribution networks. The former is the backbone of the network composed by the pipes with larger diameters, and connects six thermal plants to the various areas of the town. In the north and south plants, three cogeneration units are installed; these are able to produce up to $760 \mathrm{MW}$ of heat, as shown in Figure 1. In the transport network, water on the supply pipeline is always kept to a temperature close to the nominal temperature, i.e. about $120^{\circ} \mathrm{C}$ in winter and over 90 ${ }^{\circ} \mathrm{C}$ in summer. The temperature on the return pipeline depends on the thermal load, being about $65^{\circ} \mathrm{C}-70^{\circ} \mathrm{C}$ when the load is high and significantly reduces at night and in middle seasons or summer. As concerns pressure, values are kept above the saturation pressure values, so that there is no phase change in the network. The production capacity is completed by various heat only boilers and thermal storage units. Each distribution network links the transport network to the various buildings located in an area. Connection points between the distribution networks and the transport network are called the thermal barycenters.

The distribution network analysed here connects 11 buildings to the main pipeline. The network topology is shown in Figure 6. To solve the thermo-fluid dynamic problem in the distribution network, the knowledge of the expected thermal profile and of the mass flow rate request of each building is necessary. These pieces of information are obtained from the monitoring systems installed in the various thermal substations, roughly one in each building. Data are available for the past 4 years. Moreover, the initial conditions of the supply and return networks are computed by simulating the system shutdown of the day before the one considered, that is a typical day of January.

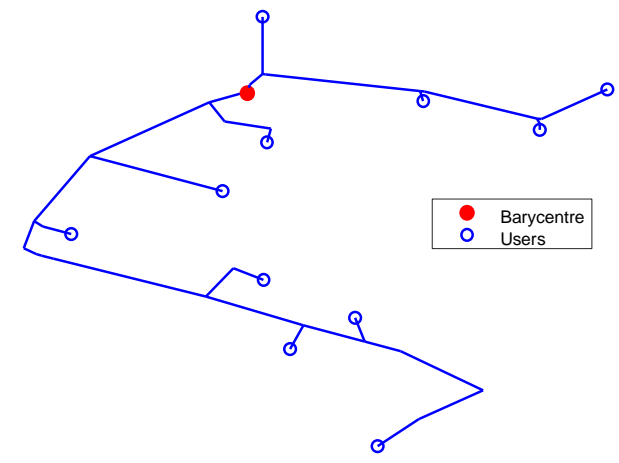

Figure 6. Schematic of the selected distribution network.

An optimization aiming at the reduction of the thermal power peak of the selected distribution network is performed. The optimization is firstly executed including in the algorithm only the return network simulation, and then both the supply and return networks simulation.

\section{Results}

By simulating the supply network it is possible to obtain the temperature values at the inlet of each user heat exchanger $\left(T_{1}\right)$. Since the thermal heat flux profile required by each building is known, it is also possible to evaluate water temperature at the outlet of each heat exchanger $\left(T_{2}\right)$. For validation purposes, these temperatures are compared with the experimental values in Figure 7 that also reports the mass flow rate required by each building.

It is possible to notice that the model is able to represent with good accuracy the temperature evolution of the network. Indeed, the only significant deviation between computational results and experimental data arises when no mass flow rate circulates in the heat exchanger. This mismatch is due to the fact that the heat losses of the heat exchanger, which are negligible when it operates, affect the temperatures registered by the sensors when it does not. When water is normally flowing, computational results are close to experimental data, proving the reliability of the model.

Figure 8 illustrates the temperature values of the water flow entering the return transport pipeline. These are obtained by applying the network model to the return distribution network. The values of the total mass flow rate are also shown in the figure. In this case, there are no experimental values available. In Figure 9, the total heat flux, computed according to Eq. (2), is reported. One can easily find the morning peak, that is about $2.7 \mathrm{MW}$. This value is about 2.5 times larger than the average thermal request during the day, which highlights the fact that there is large room for improvements. In fact, any reductions of this peak allow a better use of the network and a more efficient production of heat.

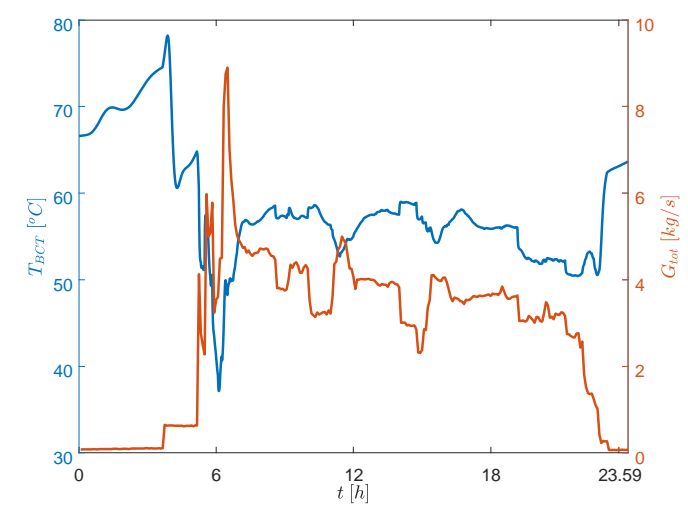

Figure 8. Temperature of water entering the return main pipeline and total mass flow rate.

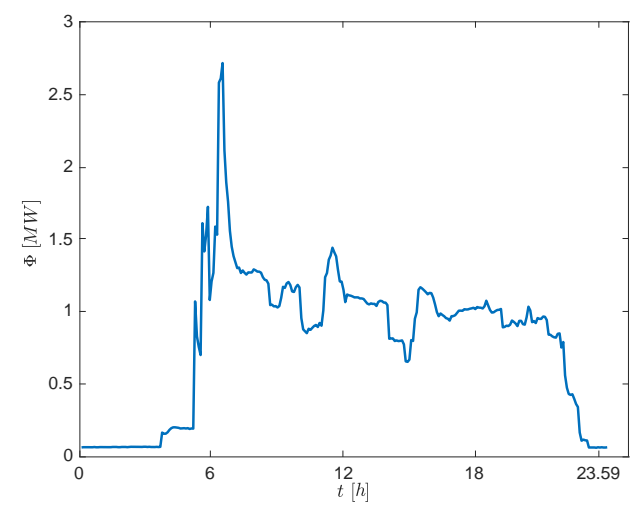

Figure 9. Heat flux required by the distribution network. 

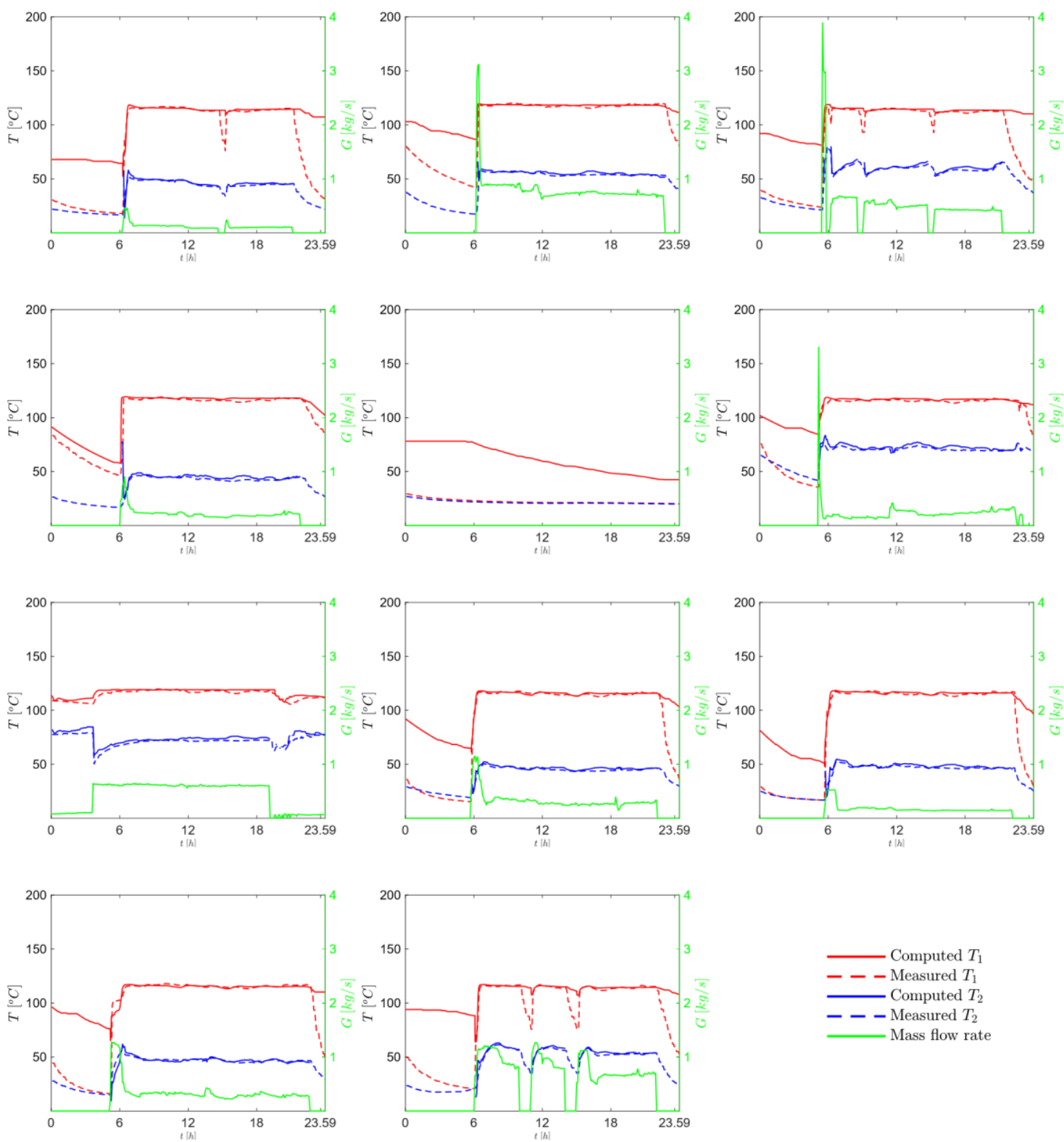

Figure 7. Temperature evolution of the water entering $\left(T_{1}\right)$ and exiting $\left(T_{2}\right)$ each user substation, both computed by the model and experimental, and mass flow rate required by each user.

The results of the optimization carried out both with the method including only the return network simulation (Figure 4(a)) and both the supply and return network simulations (Figure 4(b)) are reported in Figures 10, 11 and 12. In this case, the maximum anticipation is set to $30 \mathrm{~min}$. Method (a) gives a set of anticipations that leads to a peak reduction of $30.1 \%$ with respect to the current strategy adopted: the thermal peak is reduced to about 1.9 MW. Instead, the set of anticipations obtained with method (b) brings to a more significant reduction of the maximum thermal power, that becomes only around $1.8 \mathrm{MW}$, with a percentage reduction of $33.6 \%$ with respect to the current strategy adopted. Thus, method (b) introduces an advantage with respect to method (a): the maximum thermal power is reduced of $5.1 \%$ with respect to the previous case. Indeed, by simulating the supply network, the approximations introduced by the anticipation of the temperature of water leaving the users are no more necessary. Therefore, the model is more accurate and the optimization brings to more precise results. Moreover, the advantage obtained with the new procedure can be further improved by combining the virtual storage with other management strategies, e.g. the supply temperature control that can be only reproduced with a supply+return network simulation.

This deviation reduces when smaller anticipations are considered, which justify the use of a simpler model (i.e. return network modelling only) when small deviations are considered.

It is also important to notice that the optimal sets of independent variables using the two approaches are different. In particular, the fact that the full network is simulated involves a larger number of buildings which are anticipated of 30 minutes. This is related to the effects discussed in section 2.1 and pictorially shown in Figure 3.

Finally, the effect of the maximum allowed anticipation is analysed. The optimization is repeated considering a maximum anticipation of $15 \mathrm{~min}$ and $60 \mathrm{~min}$, respectively. Results are summarized in Figure 13. Considering a maximum anticipation of $15 \mathrm{~min}$, the peak becomes approximately $2.1 \mathrm{MW}$. The percentage reduction is thus diminished from $33.6 \%$ to $19.7 \%$. Oppositely, if the maximum anticipation is increased to $60 \mathrm{~min}$, the peak is reduced to about $1.5 \mathrm{MW}$, with a percentage reduction of $42.1 \%$. Figure 14 shows the optimal set of anticipations for each test case (in detail, it shows the number of buildings that 
are subjected to the different thermal load variations) and the modified profile of thermal request. Obviously, when the maximum allowed anticipation is increased, the potential for thermal power peak reduction increases. However, the advantage gained tends to reduce. Also, there is a need to define a trade-off between the maximum anticipation and the variations in the indoor conditions, that should be properly limited. For this purpose, the use of a building model to check the acceptability of internal temperatures may be useful, as discussed in [15]. Nevertheless, it is worth considering that anticipations make the indoor temperatures reaching the set point faster, therefore the impact on comfort conditions does not represent a real issue. Another aspect to be considered is represented by a potential increase in the total heat consumption of the buildings. This does not involve additional primary energy consumption, thanks to the larger use of cogeneration and the smaller use of boilers. In contrast, the economic impact to the end users should be carefully considered, for instance through proper incentives, in order to make such management approach a win-win strategy.

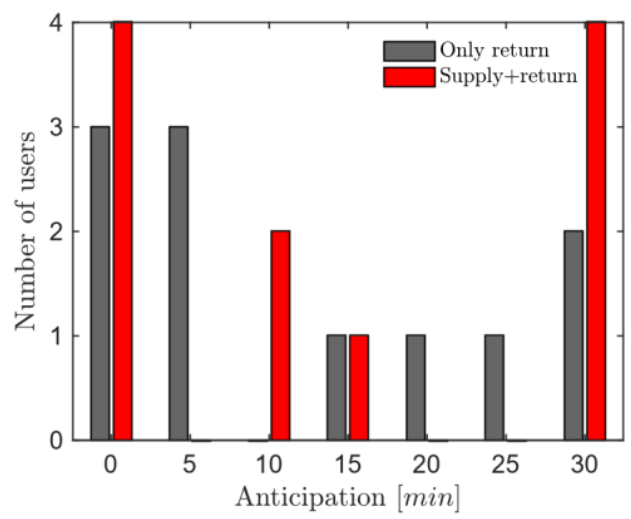

Figure 10. Optimization results: optimal set of anticipations.

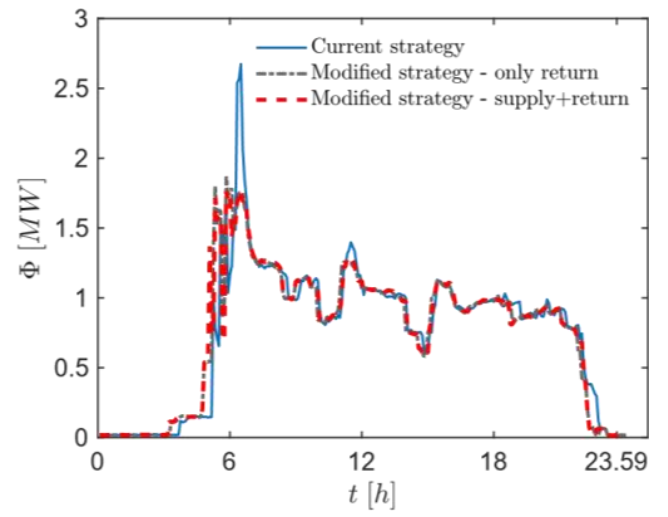

Figure 11. Optimization results: thermal power evolution.

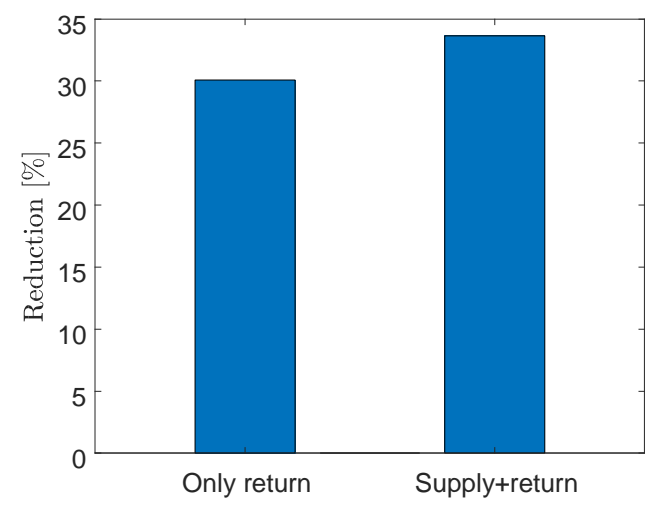

Figure 12. Optimization results: peak reduction percentage.

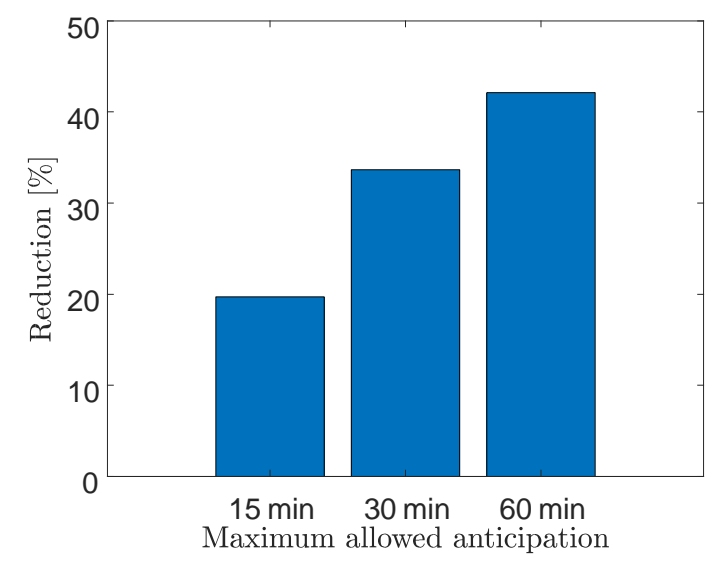

Figure 13. Percentage peak reduction for different maximum allowed anticipations.

\section{Conclusions}

In this paper, a tool for optimization of the thermal load profiles of district heating networks is presented, with the purpose of minimizing the thermal peak and improving the system efficiency. The optimization is carried out with a genetic algorithm which selects the optimal set of anticipations that the users should apply in their start-up phase. Differently from previous works, both the supply and the return networks are included in the simulations. This makes the optimization process slower but more accurate, even in the case of large anticipations. Moreover, the tool relies on a numerical method based on a more refined grid, in order to reduce the effects of artificial diffusion and to obtain a more accurate solution.

The method proposed is validated through comparison with the experimental data available for a distribution network located in Turin. With the current strategy used in this network, a thermal request peak of $2.7 \mathrm{MW}$ occurs in the morning.

For only a short time interval, the thermal power required by the distribution network is far larger than the one required most of the day. Such behaviour limits the opportunities for a full cogeneration exploitation (the use of boilers is required) and the possibility of network expansions to building or areas currently not connected.

The proposed optimization tool allows one obtaining a set of anticipations that brings to a reduction of the thermal power peak of $33.6 \%$, against the $30.1 \%$ obtained when only the return network is simulated. Different scenarios, obtained by varying the maximum anticipations are examined. In all cases, large peak reductions are achieved, between $20 \%$ and over $40 \%$. These results appear promising and suggest the application of virtual storage to the entire network.

\section{Acknowledgements}

Authors are very grateful to the technicians of IREN for providing the operation data used in this work.

\begin{tabular}{ll}
\multicolumn{2}{l}{ Nomenclature } \\
$\mathrm{A}$ & Incidence matrix \\
$c_{p}$ & Specific heat $(\mathrm{J} / \mathrm{kg} / \mathrm{K})$ \\
$\mathrm{G}$ & Mass flow rate vector $(\mathrm{kg} / \mathrm{s})$ \\
$\mathrm{g}$ & Known term accounting for losses $(\mathrm{W})$ \\
$\mathrm{G}_{\mathrm{ext}}$ & Vector of extracted/injected mass flow rates \\
& $(\mathrm{kg} / \mathrm{s})$
\end{tabular}



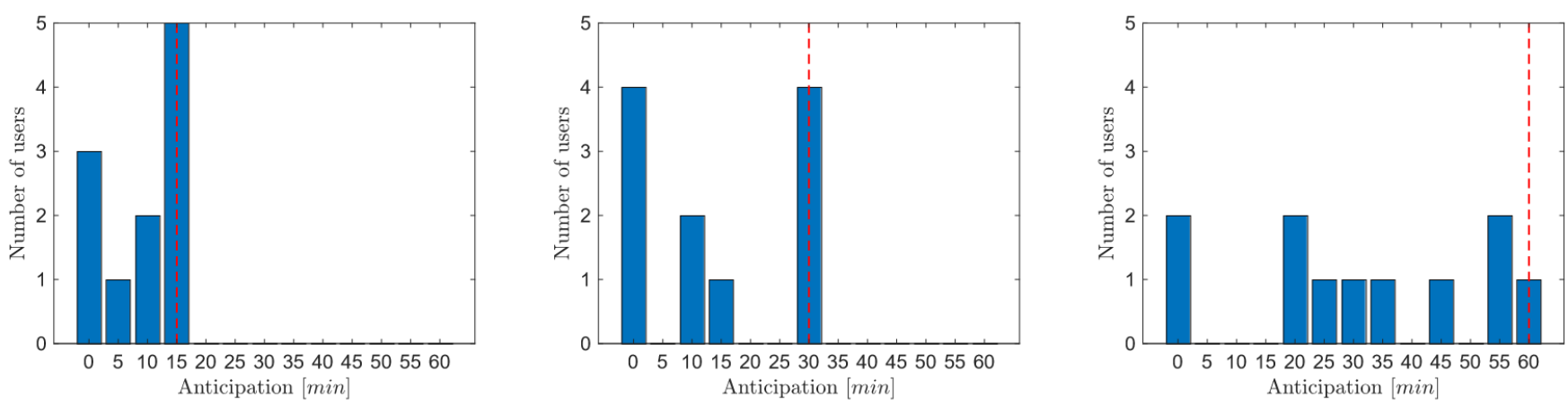

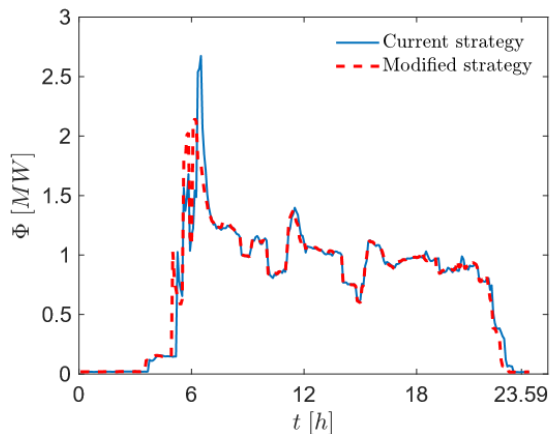

(a) $15 \mathrm{~min}$

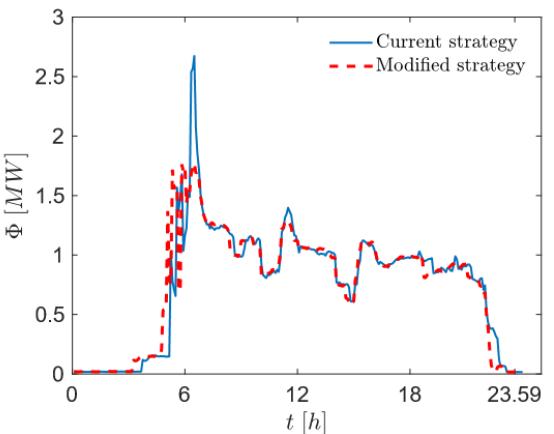

(b) $30 \mathrm{~min}$

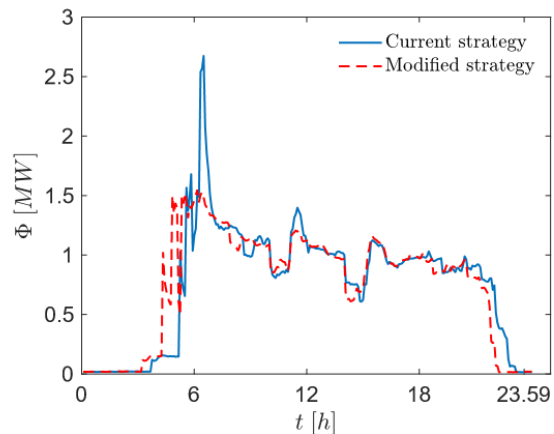

(c) $60 \mathrm{~min}$

Figure 14. Optimal set of anticipations and modified thermal request for different maximum times of anticipation allowed.

$\begin{array}{ll}G_{t o t, B C T} & \text { Total mass flow rate in the network }(\mathrm{kg} / \mathrm{s}) \\ \mathrm{K} & \text { Stiffness matrix }(\mathrm{W} / \mathrm{K}) \\ L & \text { Pipe length }(\mathrm{m}) \\ \mathrm{M} & \text { Mass matrix }(\mathrm{J} / \mathrm{K}) \\ N & \text { Number of nodes } \\ N B & \text { Number of branches } \\ O . F . & \text { Objective function }(\mathrm{W}) \\ \mathrm{P} & \text { Pressure matrix }(\mathrm{Pa}) \\ T & \text { Temperature }(\mathrm{K}) \\ T_{\text {supply }} & \text { Supply temperature }(\mathrm{K}) \\ T_{r e t u r n, B C T} & \text { Temperature of water exiting the distribution } \\ t & \text { network }(\mathrm{K}) \\ T_{\infty} & \text { Time }(\mathrm{s}) \\ U & \text { External temperature }(\mathrm{K}) \\ V & \text { Pipe transmittance }\left(\mathrm{W} / \mathrm{m}^{2} / \mathrm{K}\right) \\ v & \text { Volume }\left(\mathrm{m}^{3}\right) \\ x & \text { Velocity }(\mathrm{m} / \mathrm{s}) \\ \mathrm{X}_{\mathrm{d}} & \text { Position }(\mathrm{m}) \\ \mathrm{Y} & \text { Anticipations vector }(\mathrm{min}) \\ \mathrm{G} & \text { Fluid dynamic conductance matrix }(\mathrm{m} \mathrm{s})\end{array}$

\section{Greek symbols}

$\Gamma_{\text {num }} \quad$ Numerical diffusivity $\left(\mathrm{m}^{2} / \mathrm{s}\right)$

$\Delta x \quad$ Grid size $(\mathrm{m})$

$\rho \quad$ Density $\left(\mathrm{kg} / \mathrm{m}^{3}\right)$

$\tau \quad$ Pressure rise due to pumps vector $(\mathrm{Pa})$

$\Phi_{l} \quad$ Heat losses (W)

$\varphi_{l} \quad$ Volumetric heat losses $\left(\mathrm{W} / \mathrm{m}^{3}\right)$

$\Phi_{\text {th,tot }}$ Total thermal request (W)

$\Omega \quad$ Pipe perimeter (m)

\section{References}

[1] S. Frederiksen, S. Werner, District heating and cooling,
Lund Studentlitteratur, 2013.

[2] S. Werner, "International review of district heating and cooling”, Energy, 137, 617-631, 2017.

[3] K. Sartor, D. Thomas, P. Dewallef, “A comparative study for simulating heat transport in large district heating networks", International Journal of Heat and Technology, 36, 301-308, 2018.

[4] M. A. Ancona, F. Melino, A. Peretto, "An optimization procedure for district heating networks", Energy procedia, 61, 278-281, 2014.

[5] M. Bojic, N. Trifunovic, "Linear programming optimization of heat distribution in a district-heating system by valve adjustments and substation retrofit", Building and Environment, 35, 151-159, 2000.

[6] T. A. Koiv, A. Mikola, U. Palmiste, "The new dimensioning method of the district heating network", Applied Thermal Energy, 71, 78-82, 2014.

[7] H. Wang, W. Yin, Z. Zhou, R. Lahdelma, "Optimizing the design of a district heating network", Proceedings of ECOS 2015, Pau, France, June 30-July 31.

[8] E. Guelpa, G. Mutani, V. Todeschi, V. Verda, "Reduction of $\mathrm{CO} 2$ emissions in urban areas through optimal expansion of existing district heating networks", Journal of Cleaner Production, 204, 117-129, 2018.

[9] L. Giraud, R. Baviere, M. Vallee, C. Paulus, "Presentation, validation and application of the DistrictHeating Modelica library", Proceedings of the 11th International Modelica Conference 2015, Versailles, France, September 21-23.

[10] M. Pirouti, A. Bagdanavicius, J. Wu, J. Ekanayake, "Optimization of supply temperature and mass flow rate for a district heating network", Proceedings of ECOS 2012, Perugia, Italy, June 26-29.

[11] D. Lindenberger, T. Bruckner, H. M. Groscurth, R. Kümmel, "Optimization of solar district heating systems: seasonal storage, heat pumps, and cogeneration”, Energy, 
25(7), 591-608, 2000

[12] V. Verda, F. Colella, "Primary energy savings through thermal storage in district heating networks", Energy, 36, 4278-4286, 2011.

[13] E. Guelpa, V. Verda, "Optimization of the thermal load profile in district heating networks through "virtual storage" at building level", Energy procedia, 101, 798805, 2016.

[14] E. Guelpa, L. Marincioni, V. Verda, "Towards 4th generation district heating: Prediction of building thermal load for optimal management", Energy, 171, 510-522, 2019.

[15] V. Verda, E. Guelpa, A. Sciacovelli, A. Acquaviva, E. Patti, "Thermal peak load shaving through users request variations in district heating systems", International Journal of Thermodynamics, 19, 168-176, 2016.

[16] E. Guelpa, G. Barbero, A. Sciacovelli, V. Verda, "Peakshaving in district heating systems through optimal management of the thermal request of buildings", Energy, 137, 706-714, 2017.

[17] E. Guelpa, S. Deputato, V. Verda, "Thermal request optimization in district heating networks using a clustering approach", Applied Energy, 228, 608-617, 2018.
[18] I. Del Hoyo Arce, S. Herrero López, S. López Perez, M. Rämä, K. Klobut, J. A. Febres, "Models for fast modelling of district heating and cooling networks", Renewable and Sustainable Energy Reviews, 82, 18631873, 2018.

[19] F. Harary, Graph theory, New Delhi: Narosa Publishing House, 1995.

[20] A. Sciacovelli, V. Verda, R. Borchiellini, Numerical design of thermal systems, 2015, CLUT Editrice.

[21] E. Guelpa, A. Sciacovelli, V. Verda, "Thermo-fluid dynamic model of large district heating networks for the analysis of primary energy savings", Energy, http://dx.doi.org/10.1016/j.energy.2017.07.177.

[22] E. Guelpa, A. Sciacovelli, V. Verda, "Thermo-fluid dynamic model of complex district heating networks for the analysis of peak load reductions in the thermal plants", Proceedings of the ASME 2015.

[23] H. K. Versteeg, W. Malalasekera, An introduction to computational fluid dynamics: The finite volume method, Pearson Education Limited, 2017. 